\title{
Analysis and Simulation on Measurement Error of PLL Loop
}

\author{
Xingyuan Xu, Lili Xia \& Sheng Liang \\ China Satellite Maritime Tracking and Control Department, Jiangyin, 214431, China
}

ABSTRACT: In receiver design, how to select the frequency discriminator loop the loop filter carrier tracking loop is critical. Due to the Doppler frequency difference uncertainty exists, the carrier phase direct capture a greater degree of difficulty, but was able to capture the frequency quickly eliminate most of the effects of the Doppler shift, and then the tracking phase is relatively easy to get many. Based on this idea, this paper, the performance FLL loop analysis and simulation.

KEYWORD: FLL; measurement error; simulation

\section{INTRODUCTION}

In a spread spectrum measurement and control system, which is one of the key issues of the demodulated signal and ranging information extraction speed, so in order to ensure the receiver to work properly, the code phase (ie, code delay) and carrier frequency at the receiving end of these two uncertain time-varying parameter must be certainty. By analyzing the impact of noise on the loop FLL loop performance (Vannee D.J.R et al, 2011), and thus design suitable for high dynamic stable tracking loop bandwidth.

\section{PRINCIPLE OF PLL LOOP}

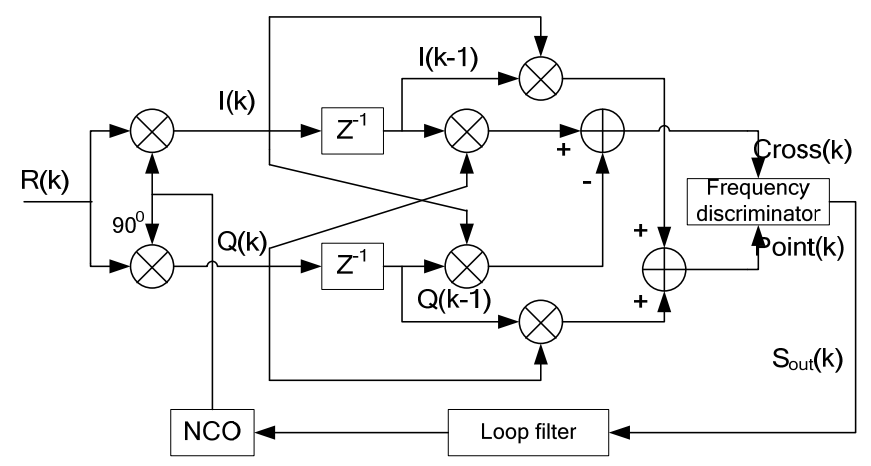

Figure 1. Block diagram of the frequency-locked loop
Frequency-locked loop (FLL) frequency tracking is essentially the difference carrier phase tracking (Van Dierendonck, 2012), which measures the amount of change in the carrier frequency discriminator in a fixed time interval is calculated based on the estimated value of the input signal Doppler frequency, its loop after adjusting filter local carrier NCO (Grant P.M et al, 2008) (Hurd W. Statman, 2007), proper frequency to demodulate the carrier signal.

Before analyzing the frequency discriminator algorithm, first introduced "dot-product" concept and "cross-product", as the frequency discriminator is on this basis to achieve. Ignore the impact of noise on the item I and Q paths. 1, set a frequency, the two signals after clearing points are:

$$
\begin{aligned}
& I(k)=A(k) \cos [\varphi(k)] \\
& Q(k)=A(k) \sin [\varphi(k)]
\end{aligned}
$$

So that

$$
\begin{aligned}
& S(k)=I(k)+j Q(k)=A(k) e^{j \varphi(k)} \\
& S_{\text {out }}(k)=S(k) S^{*}(k-1)=\operatorname{Point}(k)+j \operatorname{Cross}(k)
\end{aligned}
$$

Wherein,

$\operatorname{Point}(k)=\operatorname{Re}\left[S_{\text {out }}(k)\right]=I(k) I(k-1)+Q(k) Q(k-1) ;$ $\operatorname{Cross}(k)=\operatorname{Im}\left[S_{\text {out }}(k)\right]=Q(k) I(k-1)-I(k) Q(k-1)$. So,

$$
S_{\text {out }}(k)=A(k) A(k-1) e^{j \varphi(k)} e^{-j \varphi(k-1)}=A(k-1) A(k-1) e^{j\left[\Delta \varphi_{\bmod }(k)-\Delta \varphi_{\text {rot }}(k)\right]}
$$



able,

By equation (3) and Equation (5) Integrated avail-

$$
\begin{aligned}
\operatorname{Point}(k) & =A(k) A(k-1) \cos \left[\Delta \varphi_{\text {mod }}(k)-\Delta \varphi_{\text {rot }}(k)\right] \\
& = \pm A^{2}(k) \cos \left[\Delta \varphi_{\text {rot }}(k)\right]
\end{aligned}
$$

$$
\begin{aligned}
\operatorname{Cross}(k) & =A(k) A(k-1) \sin \left[\Delta \varphi_{\text {mod }}(k)-\Delta \varphi_{\text {rot }}(k)\right] \\
& = \pm A^{2}(k) \sin \left[\Delta \varphi_{\text {rot }}(k)\right]
\end{aligned}
$$

Wherein, $\Delta \varphi_{\bmod }(k)$ is the two successive data bits of the phase difference is $0^{\circ}$ or $180^{\circ} ; \Delta \varphi_{\text {rot }}(k)$ represents the phase difference between the local carrier and the transmission carrier; two successive sampling data bits of the accumulated values can be considered constant, i.e. $A(k)=A(k-1)$.

And PLL except that accurately reproduce the signal instantaneous phase locked loop to complete the carrier and Doppler peel extract, and frequencylocked loop is completed reproduction approximate frequency, but more PLL lock loop frequency discriminator for some I, Q two signals indeed across the phase transition of the data bits are not sensitive when compared to the pre-detection integration time and less data bit transition time interval, integral and clear affected samples It will be less. So FLL and a phase locked loop and easier compared to the frequency of the signal remains locked.

\section{DISCRIMINATOR ALGORITHM ANALYSIS}

Table 1 shows the output characteristics of the frequency error and several frequency discriminator algorithms. Figure 2 is the premise of ignoring the noise term of I and Q channel effects, the table of three frequency discriminator algorithm error output comparison.

By comparison may show that unilateral Frequency Table 1. The latter two frequency discriminator algorithm pulling range is about 2 times the frequency of a first frequency discriminator unilateral algorithm traction range. Furthermore, as the thermal noise level, amplitude discriminator output will fall

\begin{tabular}{|c|c|c|}
\hline Discriminator algorithm & $\begin{array}{l}\text { Output frequency } \\
\text { error }\end{array}$ & Features \\
\hline$\frac{\operatorname{Point}(k) \cdot \operatorname{Cross}(k)}{t_{2}-t_{1}}$ & $\frac{\sin \left[2\left(\varphi_{2}-\varphi_{1}\right)\right]}{t_{2}-t_{1}}$ & $\begin{array}{l}\text { High signal to noise ratio is close to the optimum, the slope is proportional } \\
\text { to the amplitude of the signal, a moderate amount of computation. }\end{array}$ \\
\hline$\frac{\operatorname{Cross}(k)}{t_{2}-t_{1}}$ & $\frac{\sin \left(\varphi_{2}-\varphi_{1}\right)}{t_{2}-t_{1}}$ & $\begin{array}{l}\text { Close to the best in low SNR, the slope of the amplitude of the signal pro- } \\
\text { portional to the square, the minimum amount of computation. }\end{array}$ \\
\hline$\frac{\operatorname{ATAN2}(\operatorname{Cross}(k), \operatorname{Point}(k))}{\left(t_{2}-t_{1}\right)}$ & $\frac{\varphi_{2}-\varphi_{1}}{t_{2}-t_{1}}$ & $\begin{array}{l}\text { Four-quadrant arc tangent. Close to the best estimates, the slope of the am- } \\
\text { plitude of the signal irrespective of the amount of computation demanding. }\end{array}$ \\
\hline
\end{tabular}
(slope leveled off), and the pull-in range near the edge at the start smoothing.

Table 1. Common frequency discriminator algorithm

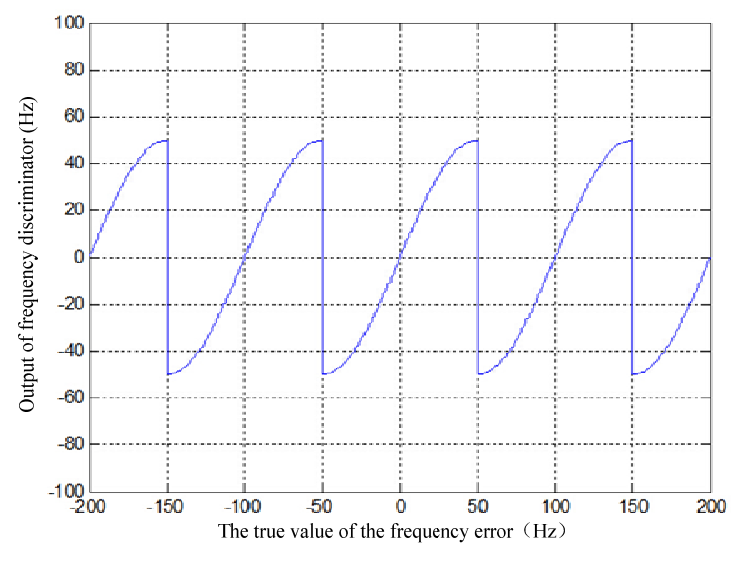

(a) $\frac{\operatorname{Point}(k) \cdot \operatorname{Cross}(k)}{t_{2}-t_{1}}$ Phase detector output

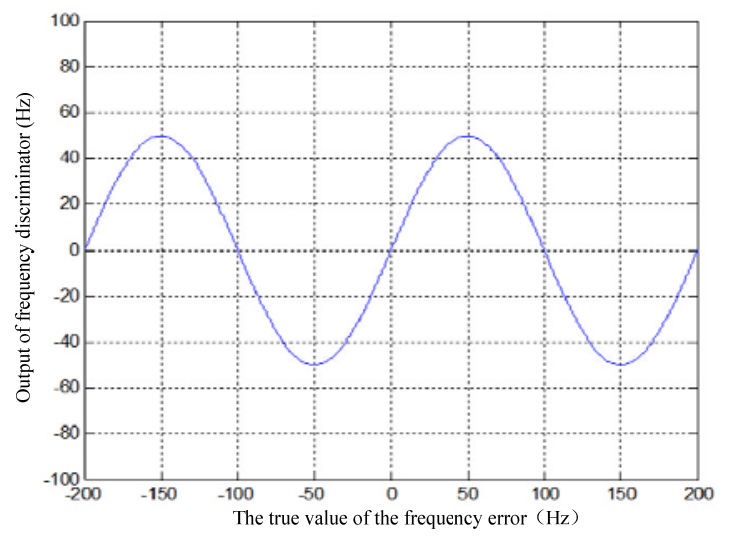

(b) $\frac{\operatorname{Cross}(k)}{t_{2}-t_{1}}$ Phase detector output 


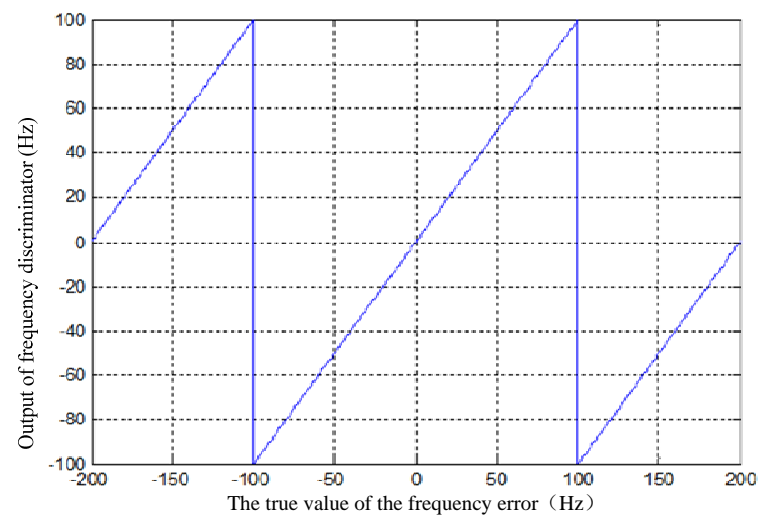

(c) $\frac{\operatorname{ATAN2}(\operatorname{Cross}(k), \text { Point }(k))}{\left(t_{2}-t_{1}\right)}$ Phase detector output

Figure 2. Comparison of frequency-locked loop frequency discriminator

\section{ANALYSIS AND SIMULATION OF MEASUREMENT ERROR}

The main source of error is the FLL frequency thermal noise frequency dither and dynamic stress error. For empirical methods FLL tracking threshold is trembling from the stress caused by all loops in a preflight integration time T must not exceed $90^{\circ}$, so the FLL tracking threshold of thumb is:

$3 \sigma_{F L L}=3 \sigma_{t F L L}+\sigma_{e F L L} \leq 0.25 / T(H z)$

Reference oscillator frequency vibration and Allan variance caused by wobbling on FLL is a small order of magnitude, is negligible. The frequency of the vibration threshold of $1 \sigma$ is $1 /(12 T)=0.0833 / T(\mathrm{~Hz})$.

FLL thermal noise caused by the tracking loop fibrillation is:

$$
\begin{aligned}
\sigma_{t F L L} & =\frac{1}{2 \pi T} \sqrt{\frac{4 F B_{n}}{C / N_{0}}\left[1+\frac{1}{T \cdot C / N_{0}}\right]}(\mathrm{Hz}) \\
\sigma_{t F L L} & =\frac{\lambda_{L}}{2 \pi T} \sqrt{\frac{4 F B_{n}}{C / N_{0}}\left[1+\frac{1}{T \cdot C / N_{0}}\right]}(\mathrm{Hz})
\end{aligned}
$$

Equation (10), when the signal to noise ratio is high, $\mathrm{F}=1$; in receiving threshold, $\mathrm{F}=2$. Figure describes the typical noise bandwidth and integration time for preflight FLL thermal noise trace fibrillation.

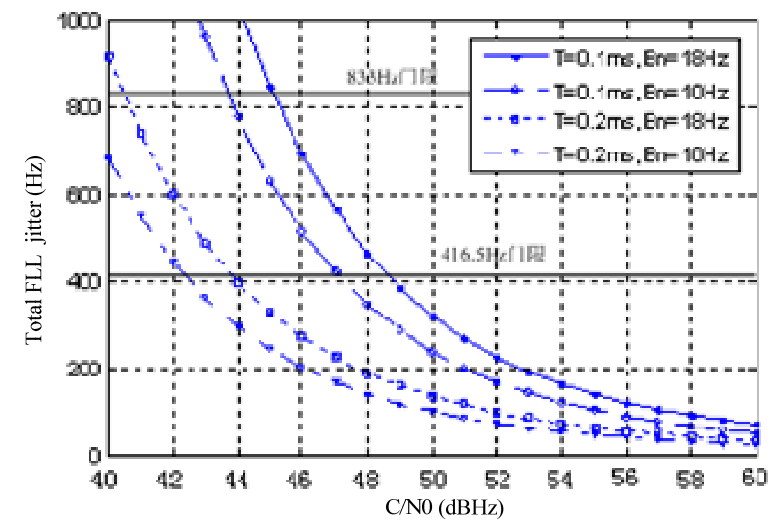

Figure 3. FLL thermal noise dither

Because tracking loop FLL same order than the PLL tracking loop one more integrator, so the dynamic stress error is:

Second-order loop:

$$
\sigma_{e F L L}=\frac{d^{3} R / d t^{3}}{360 \omega_{n}^{2}}=\frac{d^{3} R / d t^{3}}{360\left(B_{n} / 0.53\right)^{2}}=7.8 \times 10^{-4} \frac{d^{3} R / d t^{3}}{B_{n}^{2}}(\mathrm{~Hz})
$$

Third-order loop:

$\sigma_{e F L L}=\frac{d^{4} R / d t^{4}}{360 \omega_{n}^{3}}=\frac{d^{4} R / d t^{4}}{360\left(B_{n} / 0.7845\right)^{3}}=0.0013 \frac{d^{4} R / d t^{4}}{B_{n}^{3}}(\mathrm{~Hz})$

Using equation (11) simulates the dynamic relationship FLL stress threshold and noise bandwidth, shown in Figure 4:

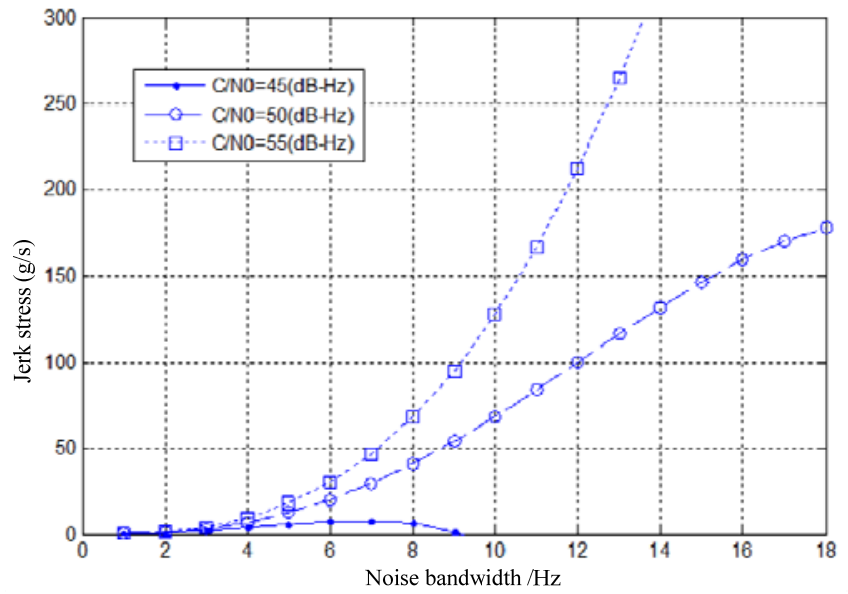

Figure 4. FLL second order dynamic relationship between stress and noise bandwidth threshold

The simulation can be found in the same situation equivalent noise bandwidth and signal to noise ratio, dynamic stress than the second order FLL thirdorder PLL good dynamic stress of about one order of magnitude, and when the FLL equivalent bandwidth is $18 \mathrm{~Hz}$ when It can be achieved on $10 \mathrm{kHz} / \mathrm{s}$ Doppler tracking the rate of change of dynamic stress. 


\section{SUMMARY}

This paper focuses on the performance of FLL loop analyzes and simulations, including the principles and FLL loop frequency discriminator algorithm by analyzing the impact of noise on the loop FLL loop performance, and thus design suitable for high dynamic stable tracking loop bandwidth.

\section{REFERENCES}

Grant P.M, Spangenberg S.M, Scott J.etal. Doppler estimation for fast acquisition in spread spectrum communication systems [J]. IEEE 2008

Hurd W.Statman. High dynamic GPS receiver using maximum likelihood estimation and frequency tracking[J]. IEEE Trans on AES July. 2007.

Van Dierendonck. GPS Receivers in Global Positioning System [J].Theory and Application.Vol.I.2012. 329 407.

Vannee D.J.R, Coenen A.J.R.M. New Fast GPS codeacquisition technique using $\mathrm{FFT}[\mathrm{J}]$. Electronics Letters.2011.27:158-161. 